\title{
The arrival of COVID-19 in the Netherlands
}

\author{
IXavier Falières
}

Anesthesiologist,

Albert Schweitzer Hospital, Albert Schweitzerplaats 25, NL-3318AT DORDRECHT, The Netherlands.

Received: 14 March 2020,

Reviewed \& Accepted: 18

March 2020

\begin{abstract}
The last three months have been the most memorable and the most tense period in the lives of the most of us. After Taking Wuhan (China) by storm, the coronavirus crossed all of the frontiers and reached to 202 countries, including the East Asian countries, Middle East, the Americas and then the European countries. The Netherlands was effected less, with about 1000 deaths and more than 12000 confirmed patients, than its neighbors - Italy and Spain, but had its share. This manuscript presents an outline of the public perceptions, and the guidelines to manage these patients at different stages of the disease, including ventilation and intubation protocols, based upon our experience of over two months. It does not claim to be complete and exhaustive, and the readers are directed to consult their National guidelines (if any).
\end{abstract}

Key words: Coronavirus; COVID-19; Mortality; Pandemic; Epidemic; Ventilation; Intubation, Endotracheal; Resuscitation; Aerosol

Citation: Falières X. The arrival of COVID-19 in the Netherlands (Perspective). Anaesth pain intensive care 2020;24(1):13-17. DOI: https://doi.org/10.35975/apic. v24i1.1220

Coronavirus COVID-19 was reported for the first time in The Netherlands on 27th February 2020, near the German border, just after Carnival festivities. The first death was notified on 6 March 2020. On 15th March, we had 1135 reported cases and 20 deaths, mostly in the South of the country. By the end of March the number of reported cases was a staggering 12,595 , and number of deaths jumped up to 1,039 .

Totally different policies to fight against this virus have been adopted within European countries. It's interesting to compare efficacy, efficiency and the results of these different approaches.

Social measures to contain the disease have gradually taken to become real drastic. Hospitals have to adapt as rapidly as the changes in the disease statistics changes at a fast pace. These sudden changes have a major impact on the population, on the economy, on patient care, and on the daily life of the Dutch people. We could only be able to measure the consequences of this dramatic period once the pandemic is finally over.

Anesthesiologists have a major role to play in this crisis. We are the ones who take care of airway, ventilation, hemodynamic status, and fight for patient's survival.
The Netherlands is separated in 2 parts by the great rivers, The North is traditionally protestant and the South (except Zealand province) is mostly catholic. In the South, Brabant and Limburg provinces had a deeply anchored Carnival tradition during the month of February. At this time of the year, skiing and enjoying holidays in Northern Italy have been popular recourse among Dutch people. The first case of COVID-19 in Northern Italy was reported on 21st February. A lot of Dutch people from the South had returned back from Northern Italy for Carnival around February 23rd. On 27th February, near the German border, the first Dutch case was reported. Then the pace of new case appearance went really fast. The Dutch mentality is to wait-and-see and not alarmist. We waited a long time before taking drastic measures. The first patient died on 6th March; and as we write these lines, the total number of deaths has crossed the figure of 1000 . If we compare to other European countries, Dutch laxity was followed by more cases, compared for instance with the very low populated Norway (5.3 million inhabitants, and a rise of total number of cases from 600 to 4600 and the deaths from 3 to 39). It seems that we don't really understand the contamination pathways.

Here is an outline of the lines of action followed by 
the Dutch people and the government.

\section{THE RULES FOR THE GENERAL POPULATION}

\section{General rules:}

- Avoid going out

- Wash your hands regularly and long enough, have a hydro-alcoholic gel with you when you are outside

- Keep a safe distance between people when possible,

- Sneeze and cough in your elbow, or use tissue paper or a piece of cloth

- Wash fruits and vegetables well, avoid buying bread or other food products that have been potentially touched by everyone and that you will not be able to clean (or toast bread before eating).

- Everyone who can work at home is invited to do it.

- Schools and universities are closed. Bars and restaurants close at $6 \mathrm{pm}$.

- The rules for quarantine are: quarantine yourself in case of cough, flu-like symptoms, fever, etc.

- Don't go to hospital or to your GP: call your GP or the GP on duty, call an ambulance if you are really feeling sick to be hospitalized.

- If you are perfectly healthy: stay home or avoid crowded places

- Keep children far away from elderly and frail people.

Many questions arise from the population:

1. Why not test everyone or everyone with symptoms? It would be a waste of time and money. It is assumed that anyone with a suspicious cough, fever or other signs of contamination should isolate themselves socially until fully recovered. Severe cases and hospital workers (doctors included) are and will be tested if needed.

2. May I work when sick? Everyone with cough or just some flu like symptoms with or without fever stay home. Hospitals: cough or just some flu-like symptoms with fever stay home

3. Why not more quarantine measures? The goal is not to stop the virus but to slow its progression. Stopping COVID-19 progression should have been a measure of isolation in the city of Wuhan on Day 1. No need to go back in time, it's too late and counterproductive. By slowing the progression of the virus, we hope for mass immunization, it gives us time for therapeutic trials. It is important to know that chloroquine has not been retained.

4. Can children be contaminated? It has been a long debate. Children and young people up to the age of 20 are certainly contaminated without any or little symptoms and contaminate us. Schools and universities are now closed.

5. What is the mortality? Definitely $1 \%$ or less. As the number of people infected is not known with certainity, the $3 \%$ mortality is no longer true except in Italy, which has decided to lock up the country with a still quite aggressive version of the virus (hypothesis of some virologists). The people who have died and who will die are mostly elderly and frail, and the less elderly but weakened by immune diseases/cancers. Then young and healthy people: like the flu pandemic, there will be deaths in young and healthy people too. There will probably be millions of deaths worldwide, we have to be prepared for that.

6. Wearing the mask? No, again, we need masks in hospitals, not everyone.

7. The fake news: how to know? Real news, fake news, rumors, conspiracy theory, are everywhere on the web. Some examples:

- Vitamins, eating well with lots of fruits and vegetables: it's common sense, but it doesn't prevent contamination.

- The story that circulates in all languages with recommendations like drinking hot water, gargling, -- uses accurate elements to preach the false.

- Coronaviruses can live long, on plastic and metal surfaces for example, it's true, hence an impeccable hand hygiene.

- Alcohol 60-85\% is effective, Chlorhexidine not mixed with alcohol is much less effective.

- Heat kills the virus: yes, but for how long? We'll see what will happen in warm countries.

- In general, coronaviruses mute and become less aggressive: this is possible, but not a generality.

- Since the beginning of 2020, there have been more than 42,000 deaths worldwide due to COVID-19 for 2 million cancer: this is true, but there is no relationship between the 2 data.

8. Medications and COVID-19. Paracetamol is indicated in case of flu-like symptoms, fever; maximum $60 \mathrm{mg} / \mathrm{kg} /$ day and no more than 3 to 4 grams per day. Corticosteroids, nonsteroidal antiinflammatory drugs (ibuprofen, diclofenac, for example), except for chronic treatment, are formally contraindicated. In case of chronic use: contact your GP.

People treated for hypertension and/or heart failure 
with ACE inhibitors (perindopril, lisinopril, Ramipril etc.) or ARB's (valsartan, candesartan, losartan, irbesartan etc.) run no risk. Don't stop your treatment. Publications reporting that these drugs could predispose to a higher risk of contamination are not based on any scientific evidence.

9. Will we have enough food? The Netherlands is the second country in the world after the USA to feed the rest of the world. If exports are limited, we will end up with an unmanageable stock of non-used food.

\section{MEASURES IN HOSPITALS}

Following principles give a general outline of the principles to be followed;

- All regular programs which can be postponed are canceled. Only onco, cardio-vascular, obstetrics, trauma, emergencies, etc. are allowed to go on.

- Nobody goes on holidays till new order. Everybody has to work on day and night shifts. All nurses, OT nurses, anesthesia nurses can help everywhere if the situation gets out of control.

- Operating rooms are divided into three categories; regular, emergency, and COVID-19 patients. One ward is dedicated to COVID-19 infected patients only.

- The ICU is divided in 3 zones: COVID-19 infected ventilated, non-infected ventilated, non-infected and non-ventilated.

- The consultations will be reduced to only what is needed. Teleconsultation are a good option. Staff could use mobile phones or hand-held walki-talkies.

- Operating rooms; At OT, we follow the European Society of Anaesthesiologists guidelines;

https://www.esahq.org/uploads/2020/03/poster-esaa3-gb.

Also from colleagues in the North of the Netherlands:

Opinions regarding intubation of patients with suspicion on or proven COVID-19

After the SARS outbreak, it was found that a significant proportion of the victims were healthcare workers. Analysis showed that a large proportion of these infections had been caused by non-timely or improper use of personal protective equipment (PPE's) and insufficient hygienic measures.

Procedures where there is a high risk of aerosol formation (body fluid of patients with virus particles in the air) are highly risky for the spread of the virus.
However, strict adherence to personal protection regulations can drastically reduce this risk.

Risky procedures in patients where aerosol may arise include:

- Manipulation of an oxygen mask

- Intubation

- Suction of sputum

Recommendations for intubation:

Based on the limited available literature, the following things can be recommended regarding intubation of a patient with COVID-19.

\section{Conditions:}

- Plan carefully in advance.

- Identify patients on time where preoxygenation, ventilation and/or intubation can be complicated.

- Give work (service) telephone to a colleague.

- Perform the procedure in an isolation room or in an operating room, preferably the room in which the patient will stay after intubation.

- The patient must wear special protective dress during transport to the location of intubation in accordance with protocol.

- Staff who have no contact should assist the patient or the bed when transporting the patient (e.g. to operate lift or manage the doors).

- All those present must bear PPE in accordance with protocol. Make sure the face mask fits properly.

- Splash glasses are necessary.

- Hand hygiene according to protocol.

- Only necessary personnel present in the room.

- Desirable: immediately outside space staff to indicate additional medication or resources.

- Consider: double gloves (inner sterile, outer nonsterile) to allow rapid glove change in contamination (e.g. by sputum).

- There are conflicting opinions regarding noninvasive ventilation. Non-invasive ventilation has been mentioned as a risk factor for aerosol production and should be avoided. According to the WHO, the latest publications suggest that non-invasive ventilation with well-fitting masks would mean lowrisk aerosol open aerosols.

- Record which individuals have been in contact with the patient.

\section{Minimizing aerosol:}

- Emergency intubation is associated with a higher 
risk of contamination. If possible, plan the intubation early.

- Respiratory management by the most experienced person.

- Careful preoxygenation through connected mask.

- Preferably with any RSI (rapid sequence intubation) avoid hood ventilation. Mask ventilation gives high chance of aerosol release.

- Modify RSI in patients where no classical RSI is possible or desirable. If mask ventilation is necessary, then use small tidal volumes.

- Just as usual in other patients, only on indication awake fiber optical intubation. Coughing and local anesthesia of the mucous membranes by lignocaine spray cause a lot of aerosol formation.

- Tracheal intubation preferably via videolaryngoscopy (Glidescope or McGrath), and not classical, because the face of the intubating person is less close to the patient's face.

- Medication: Consider intubation of an anticholinergic for the anti-salivation effect, for example glycopyrronium.

- Place used tools in a sealed bag and ensure adequate cleaning or sterilization.

- Pay attention for contamination of used oropharyngeal suction hoses.

- Use filter between tube and ventilation system when transporting an intubated patient.

\section{Resuscitation:}

- If intubation during CPR: pause chest compressions during intubation.

- Consider apnea oxygenation. Consider mask ventilation by at least two people to ensure adequate closure of the mask on the face.

- In reanimation on a ventilated patient: Go on with mechanical ventilation not manually.

- If available, use device for mechanical chest compressions to reduce risk of leakage in mask ventilation.

\section{Considerations:}

- Conservative infusion policy, unless sepsis or SIRS.

- Preferably low tidal volumes (4 to $8 \mathrm{ml} / \mathrm{kg}$ ) in low ventilation pressure with PEEP.

- Hypercapnia is allowed up to $\mathrm{pH} 7.30$ to 7.45.

\section{Intensive Care Unit:}

At the ICU, we recommend following Professor Pelosi's advice and not to use optiflow device which spreads the virus in all the room, but to intubate directly the patient.

a. Don't use noninvasive ventilation too much - if $\mathrm{SaO} 2$ less than $95 \%$ or $\mathrm{paO} 2 / \mathrm{FiO} 2$ less 200 with/ without respiratory rate higher than $25-30 \mathrm{~b} / \mathrm{min}$ at $\mathrm{FiO} 260 \%$ with CPAP by helmet after $15-30$ min - intubate immediately.

b. Low tidal volume $6-4 \mathrm{ml} / \mathrm{kg}$ body wt with minimal respiratory rate to achieve $\mathrm{pH}$ above 7.2.

c. Use relatively high PEEP at $13-15 \mathrm{~cm} \mathrm{H} 2 \mathrm{O}$.

d. Minimal alveolar recruitment strategy.

e. Lung compliance is usually good so Pplat is usually below 25-27 cmH2O (lungs are easy to ventilate) - with driving pressure below 13 $\mathrm{cmH} 2 \mathrm{O}$.

f. Increase $\mathrm{FiO} 2$ even higher than 0.5 if needed.

g. Patients usually start to have a very low paO2/ $\mathrm{FiO} 2$ when intubated - then can slightly improve - don't worry it's normal.

h. Chest x-ray usually is very bad bilaterally - use $R x$ and Echo - don't use CT scan for monitoring use echocardiography for cardiac function and pericardial effusions.

i. Use medium doses of noradrenaline, since patient are usually sedated for almost 4-7 days. It would maintain blood pressure and reduce the need for repeated visits to the patient's room.

j. Ventilation in the Prone Position is very much useful, and patients may respond well - but it should be very well-organized with considerable time required to dress the PPE before entering the patient's rooms.

$\mathrm{k}$. Be patient to weaning patients off ventilation. That should be done late. If you start that earlier, you can have problems.

1. Do a bronchoalveolar lavage (BAL) at entrance and then once weekly. Remember that swabs may show negative results while BAL shows positive results!

m. Treat patients with a cocktail of (Darunavir or Lopinavir) + Ritonavir + Oseltamivir + Hydroxychloroquine $200 \mathrm{mg}$ twice a day + Cefazoline + in addition to levofloxacin - waiting for lab results with ID team.

n. Limit IV fluids. Instead, increase noradrenaline infusion rates as needed.

o. Don't use corticosteroids, except in case of noradrenaline infusion rater higher than $0.7 \mathrm{ug} /$ $\mathrm{kg} / \mathrm{min}$, then use only hydrocortisone $50 \mathrm{mg}$ QID 


\section{perspective}

(only for very few days).

p. The use of extracorporeal membrane oxygenation (ECMO) is rare.

q. With prolonged stay in the ICU, there's an increased need of continuous renal replacement therapy (CRRT).

r. Take care of D-dimer to be measured twice a day. Increase using anticoagulation with heparin when D-dimer is increased over 4000-5000.

\section{TRIAGE}

If we can't control the situation, triage will be the next step: we will have to make choice who will be intubated and ventilated, who won't; with all the ethical questions that this raises. Should we artificially ventilate into a patient over 70 years of age, already very ill and/or demented, when we know that the chance of survival at 1 month is almost zero, or focus on those who must survive?
Conflict of interest: None claimed by the author

Author's contribution: The author accepts sole responsibility of the manuscript.

\section{Bibliography/ Further Reading:}

1. WHO: Critical preparedness, readiness and response actions for COVID-19. Available at https://www.who.int/emergencies/ diseases/novel-coronavirus-2019/technical-guidance/ critical-preparedness-readiness-and-response-actions-forcovid-19

2. Guidelines on caring for ICU patients with COVID-19. Available at https://journals.Iww.com/ccmjournal/Abstract/onlinefirst/ Surviving_Sepsis_Campaign_Guidelines_on_the.95707. aspx

3. CDC. Coronavirus Disease 2019 (COVID-19); Interim Clinical Guidance for Management of Patients with Confirmed Coronavirus Disease (COVID-19). Available at https://www. cdc.gov/coronavirus/2019-ncov/hcp/clinical-guidancemanagement-patients.htm 